\title{
Fatores associados ao clima ético em ambiente hospitalar
}

\author{
Factors associated with ethical climate in a hospital environment \\ Factores asociados con el clima ético en un entorno hospitalario
}

Recebido: 02/02/2021 | Revisado: 06/02/2021 | Aceito: 11/02/2021 | Publicado: 18/02/2021

\author{
Thaís Costa Schutz \\ ORCID: https://orcid.org/0000-0003-4341-0395 \\ Universidade Federal de Santa Maria, Brasil \\ E-mail: thais.schutz@hotmail.com \\ Taís Carpes Lanes \\ ORCID: https://orcid.org/0000-0001-9337-7875 \\ Universidade Federal de Santa Maria, Brasil \\ E-mail: taislanes_rock@hotmail.com \\ Camila Antunez Villagran \\ ORCID: http://dx.doi.org/ 0000-0002-9498-3049 \\ Universidade Federal de Santa Maria, Brasil \\ E-mail:camilaantunezvillagran@gmail.com \\ Camila Milene Soares Bernardi \\ ORCID: http://dx.doi.org/0000-0002-7213-5428 \\ Universidade Federal de Santa Maria, Brasil \\ E-mail: camilabernardi96@gmail.com \\ Graziele de Lima Dalmolin \\ ORCID: https://orcid.org/0000-0003-0985-5788 \\ Universidade Federal de Santa Maria, Brasil \\ E-mail: grazi.dalmolin@gmail.com
}

\begin{abstract}
Resumo
Introdução: Os profissionais apresentam diversos problemas relacionados à ética no ambiente de trabalho, logo, um clima ético positivo pode promover satisfação no trabalho e bem-estar social dos profissionais, tendo como objetivo identificar nas produções científicas os fatores associados ao clima ético em ambiente hospitalar. Método: Revisão integrativa realizada a partir da pergunta de pesquisa "Quais os fatores associados ao clima ético em ambiente hospitalar?" A busca foi realizada na base de dados Web Of Science, SAGE Journals Online e Nursing and Allied Health Literature, foram encontrados 399 artigos e selecionados 12 para análise. Resultados: O clima ético apresentou associação com fatores relacionados ao trabalho, como satisfação no trabalho; intensão de rotatividade; comprometimento organizacional; sofrimento moral; liderança; coragem moral; competência profissional; suporte e comportamento organizacional Discussão: Os achados mostraram que há resultados significativos e positivos em relação ao clima ético e fatores associados identificados neste estudo. Assim, apresentando resultados positivos haverá maior compatibilidade para o clima ético ser desejável no ambiente de trabalho. Conclusão: Este estudo mostra que há predomínio de associação entre clima ético e satisfação no trabalho, intensão de rotatividade, e comprometimento organizacional. Enfatiza-se a necessidade de estratégias organizacionais para proporcionar um clima ético de trabalho adequado a fim de garantir a predominância no atendimento. Além disso, é de suma importância fornecer esforços educacionais como orientação e programa de treinamento sobre ética para garantir um domínio de princípios.
\end{abstract}

Palavras-chave: Clima ético; Ética; Enfermagem; Serviços hospitalares.

\begin{abstract}
Introduction: Professionals have several problems related to ethics in the work environment, therefore, a positive ethical climate can promote job satisfaction and social well-being of professionals, aiming to identify in scientific productions the factors associated with ethical climate in the hospital environment. Method: Integrative review based on the research question "What are the factors associated with the ethical climate in a hospital?" The search was carried out in the database Web Of Science, SAGE Journals Online and Nursing and Allied Health Literature, 399 articles were found and 12 were selected for analysis. Results: The ethical climate was associated with factors related to work, such as job satisfaction; rotation intent; organizational commitment; moral suffering; leadership; moral courage; professional competence; support and organizational behavior Discussion: The findings showed that there are significant and positive results in relation to the ethical climate and associated factors identified in this study. Thus, presenting positive results, there will be greater compatibility for the ethical climate to be desirable in the work environment. Conclusion: This study shows that there is a predominance of association between ethical climate and job satisfaction, intent to turnover, and organizational commitment. Emphasis is
\end{abstract}


placed on the need for organizational strategies to provide an adequate work ethic in order to guarantee the predominance of care. In addition, it is of paramount importance to provide educational efforts such as ethics guidance and training programs to ensure a mastery of principles.

Keywords: Ethical climate; Ethic; Nursing; Hospital services.

\begin{abstract}
Resumen
Introducción: Los profesionales tienen varios problemas relacionados con la ética en el ambiente laboral, por lo tanto, un clima ético positivo puede promover la satisfacción laboral y el bienestar social de los profesionales, buscando identificar en las producciones científicas los factores asociados al clima ético en el ambiente hospitalario. Método: Revisión integradora a partir de la pregunta de investigación "Cuáles son los factores asociados al clima ético en un hospital?" La búsqueda se realizó en la base de datos Web Of Science, SAGE Journals Online y Nursing and Allied Health Literature, se encontraron 399 artículos y se seleccionaron 12 para su análisis. Resultados: El clima ético se asoció con factores relacionados con el trabajo, como la satisfacción laboral; intención de rotación; compromiso organizacional; sufrimiento moral; liderazgo; coraje moral; competencia profesional; Apoyo y comportamiento organizacional Discusión: Los hallazgos mostraron que existen resultados significativos y positivos en relación al clima ético y factores asociados identificados en este estudio. Así, presentando resultados positivos, habrá mayor compatibilidad para que el clima ético sea deseable en el ambiente laboral. Conclusión: Este estudio muestra que existe un predominio de asociación entre clima ético y satisfacción laboral, intención de rotación y compromiso organizacional. Se enfatiza la necesidad de estrategias organizativas que proporcionen una adecuada ética de trabajo para garantizar el predominio del cuidado. Además, es de suma importancia proporcionar esfuerzos educativos, tales como programas de capacitación y orientación ética para asegurar el dominio de los principios.
\end{abstract}

Palabras clave: Clima ético; Principio moral; Enfermería; Servicios hospitalarios.

\title{
1. Introdução
}

Atualmente observa-se um crescimento significativo dos interesses dos profissionais em relação ao bem-estar dos pacientes e familiares (Taraz, et al, 2019). Dessa forma, salienta-se que esses interesses promovem um processo de tomada de decisão que deve ser baseado na ética, em que é necessário por vezes confiança nas percepções individuais de cada indivíduo (Benoit, et al, 2018).

Nesse sentido, deve haver uma relação positiva entre os colegas de trabalho que necessitam de um clima seguro, para que possam discutir situações sobre o cuidado ao paciente com intuito de deliberar sobre uma situação-problema de maneira compartilhada. Logo, isso pode favorecer o aumento da confiança entre as equipes favorecendo a compreensão, trocas de conhecimentos, experiências e valores (Goo \& Sook, 2016).

A deliberação sobre dilemas éticos de forma compartilhada favorece a percepção dos profissionais sobre o clima organizacional, o qual depende do modo de gestão do trabalho. O clima ético é um tipo de clima organizacional, em que pode influenciar no comportamento dos profissionais diante da assistência e de suas relações com colegas (SCHNEIDER, et al, 2013).

Dessa forma, o clima ético pode ser definido como a perceptibilidade dos profissionais de saúde referente ao tratamento ético das questões assistenciais e das relações em seu ambiente de trabalho (Olson, 1998). Essas questões estão relacionadas aos problemas no cuidado aos pacientes e como eles são discutidos entre a equipe (Benoit, et al, 2018).

Nesse sentido, o clima pode ser classificado como positivo ou negativo, a depender das relações entre os colegas e se há embasamento em código de ética e diretrizes da instituição antes de tomar decisões sobre o cuidado ao paciente (Numminen, et al, 2015).

O clima tem sido abrangido e compreendido como um atributo da organização, em que é avaliado a partir das percepções dos trabalhadores em relação aos comportamentos padrão e estilos de gestão adotados por ela. É um fenômeno que se estabelece a partir da experiência do trabalhador, que se compõe por diferentes dimensões, compartilhado pelos integrantes de uma unidade e que tem como função orientar e regular as condutas e comportamentos individuais de acordo com os padrões determinados pelo ambiente de trabalho (Koys \& Decotiis, 1991). 
Nesse sentido, a fim de minimizar as circunstâncias no ambiente de trabalho, sugerem-se cinco tipos de clima organizacional, que podem promover uma estabilidade mais adequada no espaço laboral, sendo: profissionalismo (esperase que as pessoas sigam estritamente os aspectos legais ou profissionais), cuidar (o mais importante é o que é melhor para os outros e as pessoas zelam pelos interesses umas das outras, enquanto o objetivo principal é oferecem o maior bem para o maior número de pessoas), regras (é muito importante seguir estritamente as regras e procedimentos da organização), independente (seguimento das próprias crenças pessoais e morais), e lei e código (os profissionais devem obedecer aos regulamentos do sistema externo, como leis ou códigos de conduta profissional) (Koys \& Decotiis, 1991).

Assim, os comportamentos das organizações institucionais têm influência significativa na percepção positiva dos profissionais sobre o clima ético, a começar pela promoção de um ambiente de trabalho que favoreça o exercício de autonomia, onde possa haver discussões e tomada de decisões sobre questões que envolvam os serviços, e inclua todos os envolvidos no cuidado, como pacientes, equipes e familiares (Goo \& Sook, 2016).

Assim, a equipe de trabalho precisa manter um clima onde não haja implicações e fatores que possam causar efeitos negativos a saúde dos trabalhadores. Nesse sentido, o clima ético contribui significativamente para um ambiente saudável, onde os trabalhadores possam exercer suas atividades com satisfação e dignidade, sem que aconteça o inesperado, ou seja, sem causar fatores que implicam negativamente na saúde do trabalhador (Esmaelzadeh, 2017).

Desta maneira, o clima ético pode influenciar o bem-estar dos profissionais no espaço laboral e a qualidade da assistência prestada, o qual quando avaliado de forma negativa, pode afetar a saúde do trabalhador e a organização do trabalho. Diante disso, melhorar o espaço laboral pode ser uma estratégia eficaz para lidar com fatores associados ao clima ético, a exemplo do sofrimento moral e intenção de rotatividade, os quais são influenciados por ele.

Assim, teve-se como questão norteadora: Quais os fatores associados ao clima ético em ambiente hospitalar? Para responder a este questionamento, teve-se como objetivo geral identificar nas produções científicas os fatores associados ao clima ético em ambiente hospitalar.

\section{Metodologia}

Trata-se de uma revisão integrativa do tipo qualitativa, realizada a partir de seis etapas estabelecidas (Lacerda \& Costenaro, 2016) sendo elas:

\section{Etapa 1 - Formulação da pergunta de revisão}

A delimitação da questão norteadora "Quais os fatores associados ao clima ético em ambiente hospitalar?", aconteceu a partir da estratégia PICo: participantes (P); intervenção (I) e contexto (Co), que para esta revisão, considerouse a seguinte estrutura: P - Enfermeiros; I - Clima ético e Co - Ambiente hospitalar. Essa estratégia auxilia a encontrar a melhor evidência de forma mais efetiva e rápida (Santos, et al, 2007).

\section{Etapa 2 - Estabelecimento dos critérios de seleção e definição das bases de dados}

Os critérios de inclusão foram artigos originais completos em inglês, português ou espanhol que estivessem disponíveis na íntegra, realizados em cenário hospitalar com enfermeiros e que avaliassem a associação do clima ético com fatores relacionados ao trabalho. Foram excluídos os artigos que não respondessem à pergunta de pesquisa.

A busca foi realizada nas bases de dados: Web Of Sience, SAGE e CINAHL, utilizando-se a seguinte estratégia de busca: ("Ethical Climate" AND Nursing) com 399 estudos localizados (Web of Science, n=134; SAGE, n=173; CINAHL, $\mathrm{n}=92$ ). A busca nas bases de dados e a análise dos estudos foram realizadas em outubro de 2019 de forma duploindependente, pela autora da pesquisa e por uma bolsista de iniciação científica, previamente capacitada (Figura 1). 
Figura 1 - Fluxograma do processo de seleção duplo-independente dos artigos incluídos na revisão.

REVISOR A
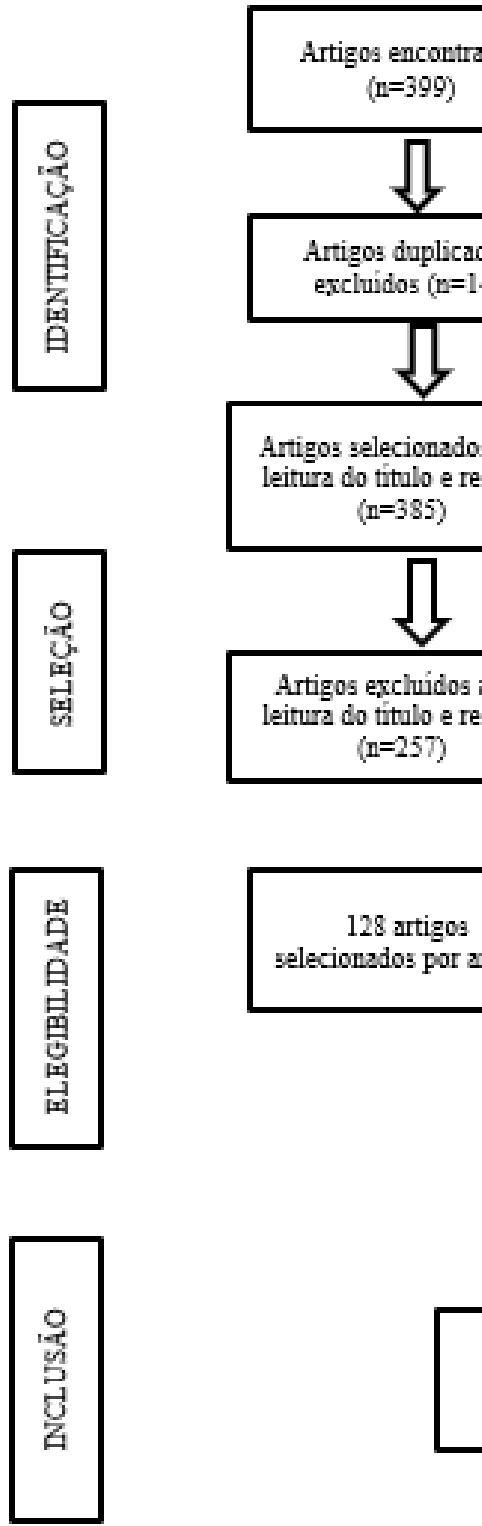
$(\mathrm{n}=385)$
REVISOR. B

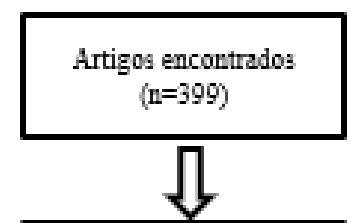

Artigos duplicados excluidos $(n=14)$

Artigos selecionados para
leitura do titulo e resumo

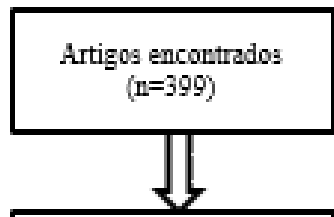

Artigos duplicado excluidos $(\mathrm{n}=14)$

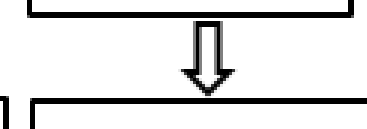

Artigos selecionados para

leitura do titulo e resumo $(\mathrm{n}=385)$

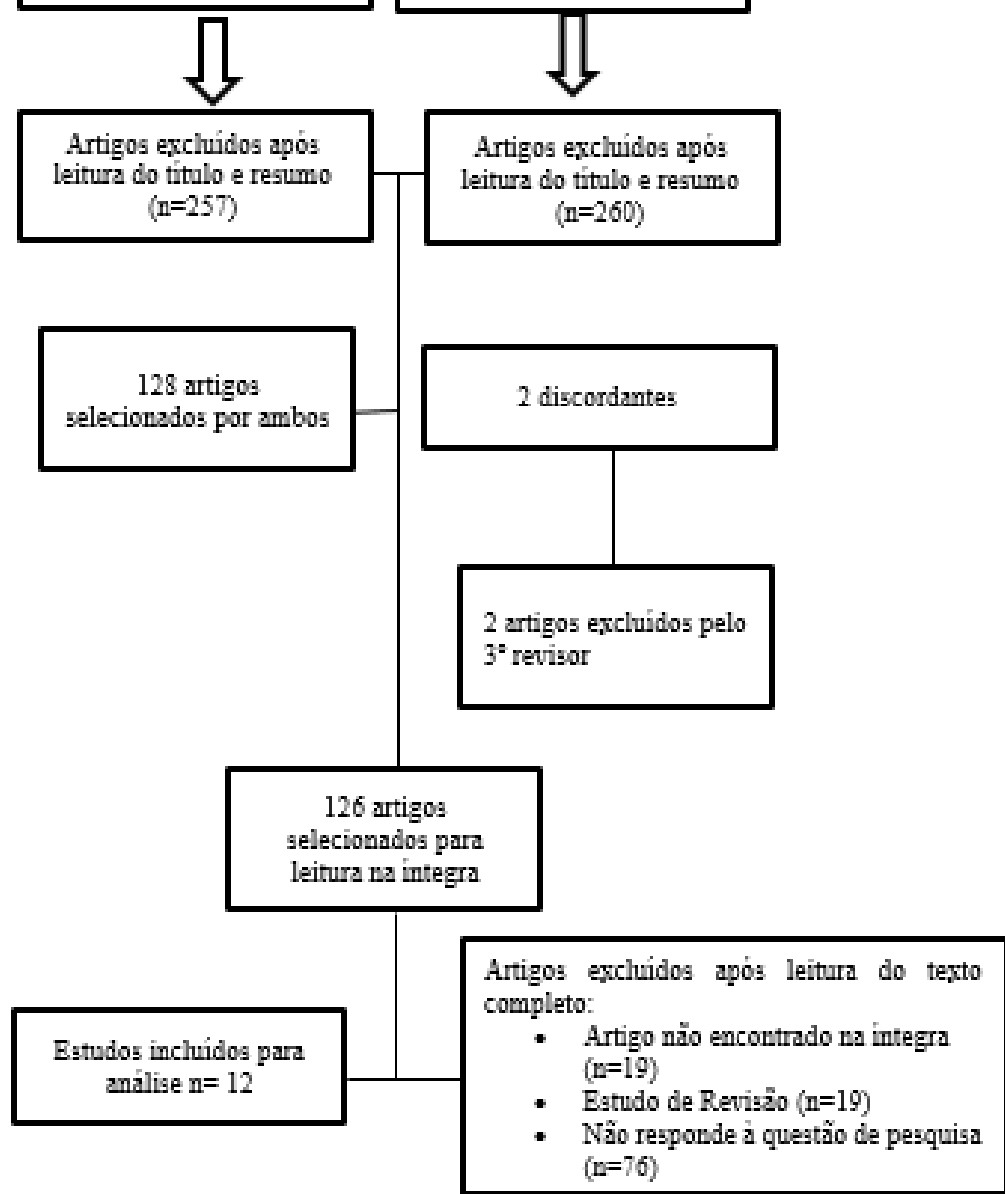

Fonte: Autores.

Os artigos encontrados em mais de uma base de dados foram incluídos apenas uma vez, restando 385 estudos para análise. Na primeira avaliação houve uma diferença de exclusão entre o revisor A e B. Diante dessa divergência de seleção, os três artigos excluídos pelo revisor B foram avaliados e excluídos por um terceiro revisor, na fase de elegibilidade. Sendo assim, permaneceram 126 estudos que após a leitura na íntegra, foram excluídos 114, obtendo-se um total de 12 artigos para a revisão. 


\section{Etapa 3 - Construção e preenchimento de um formulário para registro e extração dos dados coletados}

$\mathrm{Na}$ terceira etapa, os 12 artigos selecionados foram avaliados quanto à qualidade dos dados e sua relação com o problema de pesquisa. Para a análise, foi construída uma tabela de extração dos dados, a qual continha tais informações: autores, identificação das bases de dados, área do estudo, país e ano de publicação, periódicos, idioma, objetivos, método, resumo dos artigos, se o artigo contemplava a temática deste estudo e os principais resultados e conclusões.

\section{Etapa 4 - Avaliação crítica dos estudos primários}

A quarta etapa compreendeu a avaliação crítica dos estudos primários, por meio de um sistema de classificação de evidências. Existem diferentes classificações que podem ser utilizadas, a partir do tipo de questão de pesquisa dos estudos primários selecionados. O método escolhido foi o de classificação em sete níveis, isto é, nível 1: revisão sistemática ou metanálise de ensaios clínicos randomizados controlados; 2: ensaios clínicos randomizados controlados; 3= ensaios clínicos sem randomização; 4: coorte e caso-controle; 5= revisão sistemática de estudos descritivos e qualitativos; 6: estudo descritivo ou qualitativo; 7: opinião de especialistas; diagnóstico (um estudo primário com a questão clínica direcionada para o diagnóstico pode estar focado para determinar o quanto um teste diagnóstico é confiável para a prática clínica) (Fineout-overholt \& Melnyk, 2011).

\section{Etapa 5 - Análise e síntese descritiva dos resultados da revisão}

Esta etapa, sem sofrer inferência do revisor, possibilita analisar individualmente os estudos e a comparar os dados, com intuito de buscar resultados que possam responder à pergunta de revisão, com destaque às diferenças e similaridades entres os estudos selecionados (Lacerda \& Costenaro, 2016).

\section{Etapa 6 - Síntese do conhecimento}

$\mathrm{Na}$ sexta etapa são apresentados os resultados e conclusões dos estudos juntamente com impressões e reflexões dos autores. Ressalta-se, que a presente investigação manteve a autoria e fidedignidade dos artigos incluídos nesta revisão.

\section{Resultados e Discussão}

Predominaram estudos realizados no continente asiático $(41,67 \%$; n=5) e europeu $(33,33 \%$; n=4), publicados no periódico norte americano Nursing Ethics (75\%; n=9), entre 2009 e 2019, conforme Quadro 1. 
Quadro 1. Aspectos gerais dos estudos. (n=12). Santa Maria - RS, Brasil, 2019.

\begin{tabular}{|l|l|l|l|l|l|}
\hline \multicolumn{1}{|c|}{ ID } & \multicolumn{1}{|c|}{ Autor } & \multicolumn{1}{|c|}{ Pno } & \multicolumn{1}{|c|}{ País } & \multicolumn{1}{|c|}{ Regiãó } \\
\hline A1 & Pauly B. Et al. & 2009 & Nursing Ethics & Canadá & Norte da América \\
\hline A2 & Silén M. Et al. & 2011 & $\begin{array}{l}\text { Journal of Clinical } \\
\text { Nursing }\end{array}$ & Suécia & Norte da Europa \\
\hline A3 & Huang CC. Et al. & 2012 & Nursing Ethics & Taiwan & Ásia Oriental \\
\hline A4 & Hwang JI. Et al. & 2014 & Nursing Ethics & Coréia do Sul & Ásia Oriental \\
\hline A5 & Borhani F. Et al. & 2014 & Nursing Ethics & Irã & Sudoeste Asiático \\
\hline A6 & Numminen O. & 2015 & Nursing Ethics & Finlândia & Norte da Europa \\
\hline A7 & Dinc MS; Huric A. & 2017 & Nursing Ethics & Bósnia \\
& & & & Herzegovina & Europa Central \\
\hline A8 & Hashish EAA. & 2017 & Nursing Ethics & Egito & Norte da África \\
\hline A9 & Jang Y, Oh Y. & 2017 & Nursing Ethics & Coréia do Sul & Ásia Oriental \\
\hline A10 & Taraz, Z. Et al. & 2017 & $\begin{array}{l}\text { Iranian Red Crescent } \\
\text { Medical Journal. }\end{array}$ & Irã & Sudoeste Asiático \\
\hline A11 & Asgari S. Et al. & 2019 & Electron J Gen Med & Irã & Sudoeste Asiático \\
\hline
\end{tabular}

Fonte: Autores.

Do total de artigos, 25\% (n=3) (Pauly, et al, 2009; Huang, et al, 2012; Asgari, et al, 2019) verificaram associações significativas $(\mathrm{p}<0,05)$ do clima ético com satisfação no trabalho, comportamento organizacional e sofrimento moral. Os outros artigos, que correspondiam a 75\% (n=9) (Taraz, et al, 2019; Silén, et al, 2011; Hwang \& Park, 2014; Borhani, et al, 2014; Numminen, 2018; Dinc \& Huric, 2017; Hashish, 2017; Jang \& Oh, 2017; Ozden, et al, 2019. por meio de correlação de Pearson, identificaram as correlações que estão dispostos na Tabela 1 e 2. 
Research, Society and Development, v. 10, n. 2, e36910212577, 2021

(CC BY 4.0) | ISSN 2525-3409 | DOI: http://dx.doi.org/10.33448/rsd-v10i2.12577

Tabela 1. Correlação do clima ético com fatores relacionados ao trabalho $(\mathrm{n}=7)$.

\begin{tabular}{lll}
\hline \multicolumn{1}{c}{ Fatores } & ID & Valores de correlação \\
\hline Sofrimento Moral & A2 & $0,32^{*}$ \\
\hline Intensão de Rotatividade & A4 & $0,60^{*}$ \\
\hline Competência Profissional & A6 & $0,30^{*}$ \\
\hline Intensão de Rotatividade & & $0,10^{*}$ \\
Compromisso organizacional & A8 & $0,30^{*}$ \\
Suporte Organizacional & & $0,20^{*}$ \\
\hline & A8 & $0,20^{*}$ \\
Satisfação no trabalho & A9 & $0,66^{*}$ \\
& A12 & $0,60^{*}$ \\
\hline Coragem Moral & A10 & $0,90^{*}$ \\
\hline Liderança & A12 & $0,62^{*}$ \\
\hline
\end{tabular}

Fonte: A2, A4, A6, A8, A9, A10, A12. *Significativo para $<0,05$.

As correlações variaram de $0,1(\mathrm{~A} 8)$ a $0,90(\mathrm{~A} 11)$, em que $88,9 \%(\mathrm{n}=8)$ foram classificadas como positivas entre os fatores relacionados ao trabalho e variável clima ético.

Na Tabela 2, estão descritos a correlação entre os tipos de clima ético e os fatores do trabalho. 
Tabela 2. Tipos de Clima Ético e correlação com fatores relacionados ao trabalho (n=2).

\begin{tabular}{|c|c|c|c|c|c|c|c|}
\hline \multirow[b]{2}{*}{ ID } & Fatores & \multicolumn{6}{|c|}{ Valores de Correlação } \\
\hline & & $\begin{array}{c}\text { CE }^{* *} \\
\text { Profissionalismo }\end{array}$ & $\begin{array}{l}\text { CE** } \\
\text { Cuidar }\end{array}$ & $\begin{array}{c}\text { CE** } \\
\text { Regras }\end{array}$ & $\begin{array}{c}\text { CE** } \\
\text { Independente }\end{array}$ & $\begin{array}{c}\text { CE** } \\
\text { Instrumental }\end{array}$ & $\begin{array}{c}\text { CE** } \\
\text { Lei e códig }\end{array}$ \\
\hline A5 & $\begin{array}{l}\text { Compromisso } \\
\text { afetivo }\end{array}$ & $0,160 *$ & $0,260 *$ & $0,176^{*}$ & $0,266^{*}$ & & - \\
\hline A7 & & - & $0.253 *$ & & $-0.180 *$ & - & \\
\hline A5 & $\begin{array}{l}\text { Compromisso } \\
\text { Normativo }\end{array}$ & $0,105^{*}$ & $0,119 *$ & & & & - \\
\hline A7 & $\begin{array}{l}\text { Competência } \\
\text { profissional }\end{array}$ & - & $0.151 *$ & $0.555^{*}$ & $0.289 *$ & - & $-0.205^{*}$ \\
\hline A5 & $\begin{array}{l}\text { Compromisso de } \\
\text { continuidade }\end{array}$ & & & & & $0,221 *$ & - \\
\hline A7 & $\begin{array}{l}\text { Compromisso } \\
\text { organizacional }\end{array}$ & - & 0.106 & -0.320 & -0.108 & - & $0.381^{*}$ \\
\hline A7 & $\begin{array}{l}\text { Satisfação no } \\
\text { trabalho }\end{array}$ & - & 0.140 & $0.369^{*}$ & $0.154 *$ & - & $-0.281 *$ \\
\hline
\end{tabular}

Fonte: A5, A7 . **Clima Ético; *Significativo para $<0,05$. 
Dentre os artigos que fizeram correlação entre os fatores do trabalho com os tipos de clima ético, apresentaram correlações significativas e positivas com variação de 0,119 (5) a 0,381(7), entre eles. Os tipos de clima ético encontrados foram: profissionalismo (5), cuidar $(5,7)$, regras $(5,7)$, independente $(5,7)$, instrumental (5) e lei e código (7).

No Quadro 3, estão dispostos a identificação (Id), o delineamento de estudo (DE); os níveis de evidência (NE); os fatores associados ao CE e os principais resultados. Como DE predominou o quantitativo (100\%; n=12), com nível 6 de evidência.

Quadro 3 - Quadro Sinóptico dos artigos selecionados. Santa Maria - RS, Brasil (n=12).

\begin{tabular}{|c|c|c|}
\hline ID & $\begin{array}{l}\text { Fatores Associados ao } \\
\text { Clima Ético }\end{array}$ & Resultados \\
\hline A1 & Sofrimento moral. & $\begin{array}{l}\text { A Intensidade e a frequência de sofrimento moral foram } \\
\text { inversamente correlacionadas com as percepções do clima ético. }\end{array}$ \\
\hline A2 & Sofrimento Moral & $\begin{array}{l}\text { Houve correlação significativa entre clima ético e sofrimento } \\
\text { moral, em que quanto mais positivo o clima ético era percebido, } \\
\text { menos ocorriam situações de sofrimento moral. }\end{array}$ \\
\hline A3 & $\begin{array}{l}\text { Comportamento; } \\
\text { Comprometimento } \\
\text { organizacional. }\end{array}$ & $\begin{array}{l}\text { Há correlação negativa entre clima ético e comportamentos que } \\
\text { beneficiam a organização em geral, mas correlação positiva entre } \\
\text { clima ético e comprometimento organizacional. }\end{array}$ \\
\hline A4 & Intenção de rotatividade & $\begin{array}{l}\text { Os enfermeiros que perceberam o clima ético mais positivo para } \\
\text { os fatores "'pacientes", "gerentes", "hospital' e "médicos", logo, } \\
\text { tiveram menor intensão de deixar o local trabalho". }\end{array}$ \\
\hline A5 & $\begin{array}{l}\text { Comprometimento } \\
\text { organizacional. }\end{array}$ & $\begin{array}{l}\mathrm{O} \text { resultado desta pesquisa indicou uma correlação significativa e } \\
\text { positiva entre comprometimento organizacional e clima ético. }\end{array}$ \\
\hline A6 & $\begin{array}{l}\text { Competência profissional, } \\
\text { as intenções de rotatividade } \\
\text { e a satisfação no trabalho. }\end{array}$ & $\begin{array}{l}\text { Foram encontradas associações significativas entre o clima ético } \\
\text { percebido e a competência profissional, a intenção de rotatividade } \\
\text { e a satisfação no trabalho. Os enfermeiros com um nível de } \\
\text { competência mais alto, satisfeitos com o trabalho e com baixa } \\
\text { intensão de rotatividade perceberam o clima significativamente } \\
\text { positivo. }\end{array}$ \\
\hline A7 & Satisfação no trabalho & $\begin{array}{l}\text { Neste estudo houve correlação significativa, porém negativa entre } \\
\text { clima ético e satisfação no trabalho quando tem que seguir leis e } \\
\text { regras institucionais. }\end{array}$ \\
\hline A8 & $\begin{array}{l}\text { Satisfação no trabalho; } \\
\text { comprometimento } \\
\text { organizacional, } \\
\text { organizacional e intenção } \\
\text { de rotatividade. }\end{array}$ & $\begin{array}{l}\text { O estudo mostrou que há correlação significativa e positiva entre } \\
\text { clima ético e satisfação no trabalho, comprometimento } \\
\text { organizacional e suporte organizacional. Além disso, há correlação } \\
\text { significativa e negativa entre clima ético e intensão de } \\
\text { rotatividade. }\end{array}$ \\
\hline A9 & Satisfação no trabalho & $\begin{array}{l}\text { A satisfação no trabalho foi positivamente correlacionada com o } \\
\text { clima ético. }\end{array}$ \\
\hline A10 & Coragem moral. & $\begin{array}{l}\text { Houve uma correlação positiva e significativa entre a percepção do } \\
\text { clima ético pelos enfermeiros e sua coragem moral. }\end{array}$ \\
\hline A11 & Satisfação no trabalho & $\begin{array}{l}\text { Observou-se relação significativa entre clima ético e satisfação no } \\
\text { trabalho. }\end{array}$ \\
\hline A12 & $\begin{array}{l}\text { Satisfação no trabalho; } \\
\text { liderança }\end{array}$ & $\begin{array}{l}\text { Enfermeiros satisfeitos com o trabalho avaliaram clima ético mais } \\
\text { positivo. Apresentou correlação positiva entre o clima ético e } \\
\text { liderança ética. }\end{array}$ \\
\hline
\end{tabular}


O clima ético foi relacionado à: satisfação no trabalho (50\%; n-6); intensão de rotatividade $(33,3 \%$; n=4); comprometimento organizacional $(25 \%$; $n=3)$; sofrimento moral $(n=.2)$; liderança $(8,3 \% ; n=1)$; coragem moral $(8,3 \%$; $\mathrm{n}=1)$; competência profissional $(8,3 \% ; \mathrm{n}=1)$, suporte organizacional $(8,3 \% ; \mathrm{n}=1)$ e comportamento $(8,3 \% ; \mathrm{n}=1)$.

Quanto à caracterização dos estudos, prevaleceram as produções científicas realizadas no continente asiático e europeu, publicados entre 2009 e 2019. O que pode evidenciar que pesquisas sobre essa temática ainda são incipientes no cenário hospitalar, em especial, estudos que abordem associações do clima ético com fatores relacionados ao trabalho. No que se refere à correlação do clima ético com fatores do trabalho, verificou-se que $88,9 \%$ foram positivo entre eles.

Os tipos de clima ético encontrados nos artigos foram: profissionalismo (5), cuidar (5, 7), regras $(5,7)$, independente $(5,7)$, instrumental $(5)$ e lei e código (7), os quais apresentaram correlação significativa com os fatores do trabalho. Com relação aos tipos de clima encontrados, salienta-se que o tipo "regras" está atrelado ao que a instituição impõe, ou seja, os profissionais devem segui-las estritamente. No tipo de clima "independente”, espera-se que os indivíduos sigam suas próprias crenças pessoais e morais, cada um decide por si só. Já o "profissionalismo" apresenta-se como um seguimento de regras, ou seja, espera-se que os profissionais sigam rigorosamente as leis e normas do código de ética da profissão. O tipo "cuidar" é um dos mais importantes, pois tem por objetivo oferecer um bem-estar geral para o maior número de pessoas possível. O tipo "instrumental" objetiva fornecer benefícios pessoais, ou seja, as pessoas protegem seus próprios interesses acima de tudo e estão mais dispostas a si mesmas. Por fim, "Lei e Código" significa que os profissionais devem obedecer a regulamentos de sistemas externos (Borhani, et al, 2014; Dinc \& Huric, 2017).

Ademais, os fatores associados ao clima ético, foram: satisfação no trabalho; intensão de rotatividade; comprometimento organizacional; sofrimento moral; intenção de sair do trabalho; liderança; coragem moral; competência profissional; suporte e comportamento organizacional.

Em seis artigos (50\%) apresenta-se a satisfação no trabalho associado ao clima ético. Dessa forma, apresenta resultados favoráveis em relação ao clima ético relacionado à satisfação no trabalho, onde a qualidade no atendimento tem um aumento significativo quando se tem um clima ético positivo (Numminen, 2018). Ao estudar diferentes tipos de clima ético e relacioná-los com a satisfação no trabalho, há diversas implicações que interferem no espaço laboral de forma positiva, mas também negativa. Ou seja, o tipo de clima ético cuidar pode influenciar na satisfação no trabalho por meio de uma boa relação entre os colegas de equipe, favorecendo a qualidade da assistência. $\mathrm{O}$ tipo de clima lei e código influenciam significativamente, mas de forma negativa a satisfação no trabalho, pois os enfermeiros devem obedecer a regulamentos de sistemas externos, o que implica em sua conduta profissional (Dinc \& Huric, 2017).

Em um estudo realizado em três hospitais localizados em Taiwan com enfermeiros, correlacionou clima ético com satisfação no trabalho, identificando correlação positiva e significativa entre eles (Tsai \& Huang, 2008). Ainda, é de suma importância quando o clima ético é percebido de maneira positiva, promovendo assim um ambiente de trabalho satisfatório com alta eficácia na produtividade e relação entre equipe (Hashish, 2017; Asgari, et al, 2019). Dados esses que corroboram com diversos outros estudos, que mostram a satisfação como fator positivo quando relacionado ao clima ético. Ou seja, estar satisfeito com seu trabalho, equipe e condições gerais podem promover um ambiente favorável tanto para os pacientes e familiares quanto para equipe (Borhani, et al, 2015; Shafipour, et al, 2016; Ibrahim, et al, 2016).

Normalmente, profissionais que atuam em unidades que oferecem cuidados com maior complexidade aos pacientes, podem sentir menor satisfação com o trabalho. Por outro lado, quando há reciprocidade entre a equipe e autonomia em exercer liderança ética no setor, pode contribuir para satisfação desses profissionais, e para a percepção mais positiva do clima ético (Ozden, et al, 2019).

Em concordância, um estudo realizado em um hospital universitário da Arábia Saudita com enfermeiros, observou que os profissionais atuantes em unidades de cuidados críticos, necessitavam de maior apoio de seus líderes, para fortalecer 
a tomada de decisão. Além disso, evidenciou-se relação entre o clima ético e intensão de rotatividade (25\%), dessa forma promover ações que viabilizem o atendimento, é crucial para satisfazer o profissional no local de trabalho. Logo, a forma de trabalho deve ser prioridade para os gestores e líderes, pois assim, haverá maior confiança entre a equipe e menor intensão de deixar o trabalho (Hwang \& Park, 2014).

Para promover um atendimento de qualidade é necessário que os profissionais de saúde sejam comprometidos com o trabalho, o que pode fortalecer a percepção do clima ético. Sendo assim, o comprometimento organizacional é um fator determinante para o clima ético ser satisfatório, em que eles foram associados entre si nos estudos desta revisão (25\%; n=3) (Huang, et al, 2012; Borhani, et al, 2014; Hashish, 2017). Com isso, apresentando resultados positivos haverá maior compatibilidade para o clima ético ser desejável no ambiente de trabalho (Borhani, et al, 2014). O comprometimento organizacional indica o grau de interesse e vínculo dos funcionários em uma organização, sua aceitação de seus objetivos e valores e seu desejo de permanecer como membro dentro desta instituição e autonomia em tomar decisões com base nos preceitos éticos, o que promove um ambiente laboral mais satisfatório (Villagran, et al, 2021).

Muitos profissionais, por terem dificuldades em exercer a autonomia de solucionar e tomar decisões frente à equipe podem perceber o clima ético mais negativo, interferindo no desenvolvimento do sofrimento moral. Assim, o clima ético pode ser negativamente correlacionado com a intensidade de sofrimento, ou seja, quanto mais positivo for o clima ético, menor o nível de sofrimento moral. Porém, embora as experiências moralmente angustiantes possam não ocorrer frequentemente, elas podem ter um impacto significativo quando acontecem (Pauly, et al, 2009).

Diferente da relação do clima ético com sofrimento moral, a liderança ética tem correlação significativa e positiva com o clima ético. Além disso, evidenciou- se a importância da liderança ética interligada ao clima ético, fundamentandose em fatores positivos como condições de trabalho e ligação entre as equipes e seus líderes (Ozden, et al, 2019). Em meio a isso, sabe-se que enfermeiros frequentemente resolvem problemas com base em diretrizes e códigos de ética, no entanto, na maioria das vezes são impostas restrições que os obrigam a comprometerem e divergirem dos seus valores diante das tomadas de decisão (Silén, et al, 2012).

Além disso, a coragem moral pode ser um fator determinante que auxilia nas ações para melhorar a convivência no espaço laboral. Portanto, é necessário que a gestão hospitalar, assim como toda a equipe esteja envolvida no processo do cuidado, mostrando o quão importante é a maneira de lidar com os pacientes que buscam ajuda profissional. Dessa forma, relacionar e abordar questões éticas possibilita a compreensão de informações gerais, que por vezes são aplicadas nas ações do clima ético que tende a ser positivo (Taraz, et al, 2019).

Assim, ressalva-se ainda, que o profissional deve ser capaz de atuar integralmente no local de trabalho aplicando, conhecimentos, habilidades, atitudes e valores em uma determinada situação contextual de trabalho abrangendo sua competência profissional (Numminen, 2015). Vários estudos apontam a competência profissional como um fator essencial para melhor qualidade no atendimento, porém, são poucas as pesquisas que mencionam a relação entre clima ético e competência profissional (Numminen, 2018; Walker \& Campbell, 2013). Ainda, estudos têm indicado que a competência profissional está associada às intenções de rotatividade e satisfação do trabalho. A disposição dos profissionais em mudar de emprego ou deixar a profissão é alarmante, e isso pode interferir em sua competência profissional, uma vez que esses não realizam suas tarefas adequadamente quando não há satisfação e um clima ético positivo (Walker \& Campbell, 2013).

Dessa forma, deve haver um suporte organizacional para melhorar o rendimento do profissional. Ainda, a relação positiva entre clima ético e suporte organizacional promove um atendimento de qualidade, ou seja, os profissionais realizam seu trabalho em prol da saúde dos pacientes e esses ficam satisfeitos com o atendimento (Hashish, 2017). Além disso, aumenta o nível de satisfação quando há apoio institucional aos funcionários, consequentemente haverá um clima 
ético positivo, o que poderá promover o sucesso profissional além da redução de estresse e negatividade no trabalho. Assim, ocorrerá excelência moral e aumento da produtividade através de práticas éticas no espaço laboral (Nafei, 2015).

Além disso, comportamentos ideais promovem atendimento desejável e sem restrições, dessa forma, a atenção do profissional intensifica a qualidade do atendimento em saúde (Borhani, et al 2014). Esse achado concorda com os resultados de um estudo em que existe uma correlação positiva e significativa entre comportamento e clima ético, o que significa que quanto maior o comprometimento organizacional dos enfermeiros, mais positivo o clima ético (Villagran, et al, 2021). Além disso, o clima ético também apresenta correlação significativa e positiva quando diz respeito ao comprometimento de cada profissional e seu ambiente de trabalho. Ou seja, as ações dos profissionais podem interferir no clima ético, transformando assim, o ambiente laboral da equipe que realiza as ações em prol dos pacientes (Hashish, 2017).

Entende-se que o cuidado ao paciente começa desde a recepção até a parte assistencial, com isso é fundamental haver práticas que corroborem o bem-estar dos profissionais. Dessa forma, é necessário existir orientações e atividades em grupo que reforcem o objetivo em comum, baseando-se em conceitos éticos que comprovem a satisfação dos profissionais.

Percebe-se ainda, existir a necessidade de conscientização por parte dos profissionais em relação à essencialidade no atendimento em equipe. Assim, torna-se fundamental abordagem nas atividades em grupo sobre valores pessoais, éticos e morais, a fim de refletirem questões interpessoais e no atendimento ao paciente. Dessa forma, haverá o surgimento de novas ideias e saberes, que, por conseguinte influenciará diretamente no resultado do nível de clima ético.

Os fatores que influenciam o clima ético podem ocasionar diversas mudanças no ambiente de trabalho. Dessa forma, torna-se imprescindível a busca por um local onde haja constante harmonia entre os profissionais. Assim, haverá maior contribuição para um clima ético positivo e favorável.

\section{Conclusão}

Os resultados deste estudo mostraram que existe uma relação positiva entre clima ético com sofrimento moral; liderança; coragem moral; competência profissional; suporte e comportamento organizacional, em que predominou a associação entre o clima ético e satisfação no trabalho, intensão de rotatividade e comprometimento organizacional.

Os resultados indicaram que o clima ético favorável é de suma importância para um atendimento de qualidade, dessa forma, obter meios para que a equipe sinta-se satisfeita com seu trabalho é crucial para o atendimento. Os gerentes de enfermagem devem prestar atenção a fatores que melhoram o clima ético. Portanto, recomenda-se que para pesquisas futuras sejam realizadas ações que visem à promoção do clima ético no contexto hospitalar, com vistas a promover um ambiente ético e seguro para os pacientes e profissionais de saúde.

\section{Referências}

Asgari, S., et al. (2019). Relationship between moral distress and ethical climate with job satisfaction in nurses. Nursing Ethics. 26(2): 346-356. https://doi.org/10.1177/0969733017712083

Benoit, D. D., et al , (2018). Outcome in patients perceived as receiving excessive care across diferente ethical climates: a prospective study in 68 intensive care units in Europe and the USA. Intensive Care Med. 44: 1039-1049. https://doi.org/10.1007 / s00134-018-5231-8

Borhani, F., et al, (2015). Nurses' perceptions of ethical climate governing the teaching hospital affiliated with the University of Medical Sciences Shahid Sadughi Yazd. Med Ethics. 8(29): 41-65.

Borhani, F., et al (2014). Nurses' perception of ethical climate and organizational commitment. Nursing Ethics. 21(3): 278-288. https://doi.org/10.1177/0969733013493215

Dinc, M. S. \& Huric, A., (2017). The impact of ethical climate types on nurses' behaviors in Bosnia and Herzegovina. Nursing Ethics. 24(8): 922-935. https://doi.org/10.1177/0969733016638143 
Villagran, C. A., et al, (2021). Trends in brazilian scientific productions about ethical issues and worker's health. Research, Society and Development, 10(1): e5010111258. Doi: 10.33448/rsd-v10i1.11258.

Esmaelzadeh, F., (2017). Ethical Leadership and Organizational Climate: The Experience of Iranian Nurses. Iran Red Crescent Med J. 19(4): e43554. https://doi.org/10.5812/ircmj.43554

Fineout-overholt, E. \& Melnyk, B. M., (2011). Evidence-based practice in nursing \& healthcare. A guide to best practice. Philadelphia: Wolters Kluwer, Lippincott Williams \& Wilkins.

Goo, N. Y. \& Sook, J. M., (2016). Path analysis of the Influence of Hospital Ethical Climate Perceived by Nurses on Supervisor Trust and Organizational Effectiveness. J Korean Acad Nurs. 46(6): 824- 835. https://doi.org/10.4040/jkan.2016.46.6.824

Hashish, E. A. A., (2017). Relationship between ethical work climate and nurses' perception of organizational support, commitment, job satisfaction and turnover intente. Nursing Ethics. 24(2): 151-166. https://doi.org/10.1177/0969733015594667

Huang, C. C., et al, (2012). A multidimensional analysis of ethical climate, job satisfaction, organizational commitment, and organizational citizenship Behaviors. Nursing Ethics. 19(4): 513-529. https://doi.org/10.1177/0969733011433923

Hwang, J. I. \& Park, H. Á., (2014). Nurses' perception of ethical climate, medical error experience and intent-to-leave. Nursing Ethics. 21(1): 28-42. https://doi.org/10.1177/0969733013486797

Ibrahim, N. K., et al, (2016).. Quality of life, job satisfaction and their related factors among nurses working in king Abdulaziz University Hospital, Jeddah, Saudi Arabia. Contemporary nurse. 52(4): 486-498. https://doi.org/ https://doi.org/10.1080/10376178.2016.1224123

Jang. Y. \& Oh, Y. (2017). Impact of ethical factors on job satisfaction among Korean nurses. Nursing Ethics. 1(13). https://doi.org/10.1177/0969733017742959

Koys, D. J. \& Decotiis, T. A. (1991). Inductive measures of psychological climate. Human Relations. 44(3): 265-285. https://doi.org/10.1177/001872679104400304

Lacerda, R. M. \& Costenaro, R. G. S. (2016). Metodologia da pesquisas para a enfermagem e saúde: da teoria à prática. 1. ed. Porto Alegre: Moriá.

Nafei, W. A. (2015). Ethical climate as a moderator of the relationship between job satisfaction and organizational commitment: evidence from industrial companies in Egypt. Int J Invest Manag Financ Innov. 1(1): 1-15.

Numminen, O., et al, (2015). Ethical climate and nurse competence - newly graduated nurses perceptions. Nursing Ethics. 22(8): 845-859. https://doi.org/10.1177/0969733014557137" It "_blank

Numminen, O. (2015). Ethical climate and nurse competence - newly graduated nurses' perceptions. Nursing Ethics. 22(8): 845-859. https://doi.org/10.1177/0969733014557137

Olson, L. L. (1998). Hospital nurses' perceptions of the ethical climate of their work setting. Image J Nurs Sch. 30: 345-9. https://doi.org/10.1111 / j.15475069.1998.tb01331.x

Ozden, D., et al, (2019). The effect of nurses' ethical leadership and ethical climate perceptions on job satisfaction. Nursing Ethics, 26(4): $1211-1225$. https://doi.org/10.1177/0969733017736924

Pauly, B., et al, (2009). Registered Nurses' Perceptions of Moral Distress and Ethical Climate. Nursing Ethics. 16(5): 561-573. https://doi.org/10.1177/0969733009106649

Sajjad, M. O. S. Q. R. (2015). Emotional exhaustion and its correlation with job performance and job satisfaction in the Kingdom of Saudi Arabia. Mediterr J Soc Sci. 6(3): 51-62. https://doi.org/10.5901/mjss.2015.v6n3s1p51

Santos, C. M. C., et al (2007). Estratégia PICO para construção da pergunta de pesquisa e busca de evidências. Rev. Latino-am Enfermagem. 2007; 15(3). https://doi.org/10.1590/S0104-11692007000300023

Schneider, B., et al, (2013). Organizational climate and culture. Annu Rev Psychol. 64:361-88. https://doi.org/10.1146/annurev-psych-113011-143809

Shafipour, V., et al (2016). Nurses' perception of the ethical climate in the Iranian hospital environment. Iran J Nurs Midwifery Res, 3(4): 37-43. https://doi.org/10.18869 /acadpub.jnms.3.4.37

Silén, M., et al, (2011). Moral distress and ethical climate in a Swedish nursing context: perceptions and instrument usability. Journal of Clinical Nursing. 20: 3483-3493. https://doi.org/10.1111/j.1365-2702.2011.03753.x

Taraz, Z., et al, (2019). The relationship between ethical climate of hospital and moral courage of nursing staff. Electron J Gen Med. 16(2). https://doi.org/10.29333/ejgm/93472

Tsai, M. T., \& Huang, C. C. (2008). The Relationship among Ethical Climate Types, Facets of Job Satisfaction, and the Three Components of Organizational Commitment: A Study of Nurses in Taiwan. Journal of Business Ethics. 80:565-581. https://doi.org/10.1007/s10551-007-9455-8

Walker, A., \& Campbell, K. (2013).Work readiness of graduate nurses and the impact on job satisfaction, work engagement and intention to remain. Nurs Educ Today. 33: 1490-1495. https://doi.org/10.1016/j.nedt.2013.05.008 